\title{
Intersection Number of Paths Lying on a Digital Surface and a New Jordan Theorem
}

\author{
Sébastien Fourey and Rémy Malgouyres \\ GREYC, ISMRA, 6, bd Maréchal Juin 14000 Caen \\ \{Fourey, Malgouyres\}@greyc.ismra.fr
}

\begin{abstract}
The purpose of this paper is to define the notion of "real" intersection between paths drawn on the $3 \mathrm{~d}$ digital boundary of a connected object. We consider two kinds of paths for different adjacencies, and define the algebraic number of oriented intersections between these two paths. We show that this intersection number is invariant under any homotopic transformation we apply on the two paths. Already, this intersection number allows us to prove a Jordan curve theorem for some surfels curves which lie on a digital surface, and appears as a good tool for proving theorems in digital topology about surfaces.
\end{abstract}

Key words: Digital topology, digital surfaces, surfels curves.

\section{Introduction}

Digital surfaces of three dimensional objects have proved to be a fruitful model for visualisation and analysis of the objects they represent ([2]), especially in the biomedical field. Efficient algorithms for extracting surfaces from volumes, and computing shape characteristics exist (4]). Sometimes, the surface itself needs to be segmented since some anatomic points are defined on it. Then we obtain some subset $X$ of the set of the surfels of the surface. In [5], several tools of image analysis are defined in this nonplanar framework to analyse the topology of the subset $X$.

In particular, in [5], two complementary adjacency relations between surfels on a digital surface are introduced, the $e$-adjacency and the $v$-adjacency, and a notion of topology preservation is proposed. Given $Y \subset X$ two subsets of a digital surface, a relationship is established, between the property of $X$ to be reducible to $Y$ by sequential deletion of simple surfels, and the fundamental groups ([3]) of $Y$ and $X$. However, the conditions obtained in 5] seem redundant, and the authors conjecture that a stronger theorem exists. Such powerful results are now difficult to prove because of the lack of tools for proving theorems about homotopy of closed paths.

On the other hand, topological properties of curves, well known in the $2 D$ planar discrete case, have not yet been studied for curves on digital surfaces. However, the nonplanar framework imposes us to take into account the notion 
of homotopy properties of the considered curves. Again, the need for the development of a tool for proving theorems about homotopy of curves or closed paths appears.

The purpose of this paper is to propose such a tool. Given two paths $c$ and $\pi$ on a digital surface, we define a nontrivial integer invariant $\mathcal{I}_{\pi, c}$, called the intersection number of $\pi$ and $c$, an $e$-path and a $v$-path, which represents intuitively the algebraic number of transverse intersections between $c$ and $\pi$, summing algebraically the intersections with a sign depending on their orientations. We prove that the number $\mathcal{I}_{\pi, c}$ is invariant when $c$ or $\pi$ ranges within a homotopy class of paths. In other words, the number $\mathcal{I}_{\pi, c}$, which can be computed with the data of $c$ and $\pi$, is in fact a function of the homotopy classes of $c$ and $\pi$.

This invariant can for instance be used as follows: given a closed path $\pi$, we can prove that $\pi$ is not homotopic to a trivial path by exhibiting another path $c$ such that $\mathcal{I}_{\pi, c} \neq 0$. We illustrate this principle by proving a Jordan property for those of the simple closed curves which are homotopic, as closed paths, to a trivial path. The intersection number appears as a good tool for proving theorems in digital topology, and we expect to use it in the future to prove more results, in particular concerning topology preservation within surfaces.

\section{Basic notions and definitions}

To define the notion of a digital surface, we must recall few notions of digital topology. First, we consider objects as subsets of the 3 dimensional space $Z^{3}$. Elements of $\mathrm{Z}^{3}$ are called voxels (short for "volume elements"). Voxels which do not belong to an object $O \subset \mathrm{Z}^{3}$ constitute the complement of the object and is denoted by $\bar{O}$. Any voxel can be seen as a unit cube centered on a point with integer coordinates : $v=(i, j, k) \in \mathrm{Z}^{3}$. Now, we can define some binary symetric antireflexive relations between voxels. Two voxels are said 6 -adjacent if they share a face, 18-adjacent if they share an edge and 26-adjacent if they share a vertex. For topological considerations, we must always use two different adjacency relations for an object and its complement. We sum this up by the use of a couple $(n, \bar{n})$ with $\{n, \bar{n}\}=\{6,18\}$, the $n$-adjacency being used for the object and the $\bar{n}$-adjacency for its complement. By transitive closure of these adjacency relations, we can define another one : connectivity between voxels. We define an $n$-path $\pi$ with a length $k$ from a voxel $a$ to a voxel $b$ in $O \subset \mathrm{Z}^{3}$ as a sequence of voxels $\left(v_{i}\right)_{i=0 \ldots k}$ such that $\forall 0 \leq i<k, v_{i}$ is $n$-adjacent or equal to $v_{i+1}, v_{0}=a$ and $v_{k}=b$. Connectivity now can be defined; two voxels $a$ and $b$ are called $n$-connected in an object $O$ if there exists an $n$-path $\pi$ from $a$ to $b$ in $O$. This is an equivalence relation on voxels of an object $O$, and the $n$-connected components of the object $O$ are equivalence classes of points according to this relation. Using this equivalence relation on the complement of an object we can define a background component of $O$ as a connected component of $\bar{O}$. 


\subsection{Digital surface}

In this paper, we are interested by surfaces constituted by the boundary between a 6-connected or 18-connected subset $O$ of $\mathrm{Z}^{3}$ and $V$, one of its background components. As in 7] we first define the border between $O$ and $V$ by:

$$
\delta(O, V)=\{(a, b) \mid a \text { is } 6 \text {-adjacent to } b, a \in O, b \in V\}
$$

The set $\Sigma=\delta(O, V)$ is called a digital surface and has the Jordan property (according to the definition given in [7]).

Each couple $(a, b)$ of $\Sigma$ is called a surfel (short for surface element) and can be seen as the common face shared by two 6 -adjacent voxels, the first one belonging to the object, the second one to the background. Note that such a face is oriented according to the outward normal and this definition of a surfel is more restrictive than the classic one. In fact, we call a voxel face the unit square shared by any two 6-adjacent voxels, but a surfel is the oriented common face of two 6-adjacent voxels, where the first on is a voxel of $O$ and the second one a voxel of $V$.

In the sequel of this paper, $\Sigma=\delta(O, V)$ is a digital surface.

\subsection{Surfels Neighborhood}

A surfel in a digital surface shares a given edge with at most three other ones. Depending on the adjacency considered for the object (6 or 18), we can define an adjacency relation between surfels in such a way that a surfel has exactly four neighbors, one per edge (whereas at most 3 other surfels can share a given edge). The definition of this classical regular graph on $\Sigma$ can be found for instance in [6]. This adjacency is called $e$-adjacency (short for "edge adjacency"). As in [5] we define a loop as an $e$-connected component of the set of the surfels which share a given vertex (see Figure 1). One can see that a vertex is not sufficient to uniquely define a loop since a vertex can define two distinct loops. In fact, a loop is well defined given a vertex and a surfel incident to this vertex.

We say that two surfels are $v$-adjacent (short for "vertex adjacent") if they belong to a common loop. We denote by $N_{n}(x)$ for $n \in\{e, v\}$ the $n$-neighborhood of the surfel $x$, i.e. the set composed of the surfels of $\Sigma$ which are $n$-adjacent to $x$.

In the case when $O$ is considered as 18 -connected, we avoid some special configurations by the assumption that any loop of the surface is a topological disk. A formal way to express this assumption is to say that two $v$-adjacent surfels which are not $e$-adjacent cannot both belong to two distinct loops. An equivalent formulation can be stated as follows: we assume that if the object $O$ the surface of which we consider is studied with 18-connectivity, and if there exists in $O$ two 18-adjacent voxels which are not 6-adjacent (see Figure 2) then, at least one of the two following properties is satisfied:

- The two voxels have an 18-neighbor in $O$ in common.

- The voxels have two 26-neighbors in $O$ in common which are themselves 26-adjacent. 


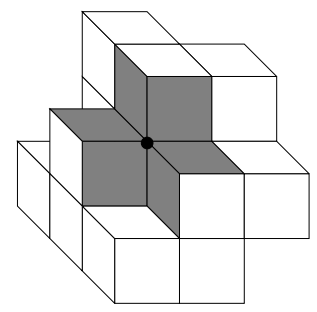

Fig. 1. Example of a loop.

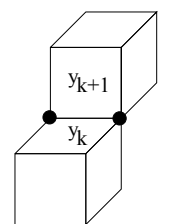

Fig. 2. A pathological case for which a loop is not a topological disk.

This restriction is necessary and sufficient to ensure that a loop is a topological disk. However, given a surfel $x \in \Sigma$, we will also need to define a topology on the neighborhood $N_{v}(x)$ under which it is a topological disk too (see [5]). For this purpose, we define the $e_{x}$-adjacency relation:

Definition 1 Let $x$ be a surfel of a digital surface $\Sigma$. We say that two surfels $y$ and $y^{\prime}$ of $N_{v}(x) \cup\{x\}$ are $e_{x}$-adjacent if they are $e-$ adjacent and both contained in a loop which contain $x$.

This relation allows us to deal with the $e_{x}$-connected component of $N_{v}(x) \cap X$ where $X$ is a set of surfels.

\subsection{Surfels paths}

Let $n \in\{e, v\}$.

Definition 2 An $n$-path $c$ with a length $l$ in a digital surface $\Sigma$ is a sequence $\left(s_{k}\right)_{0 \leq k \leq l}$ of $l+1$ surfels for which $s_{k}$ is $n$-adjacent or equal to $s_{k+1}$ for $0 \leq k<l$. $A$ closed $n$-path is a path such that $s_{l}=s_{0}$.

Notation 1 For a sequence $s=\left(s_{i}\right)_{0 \leq i \leq n}$ of surfels, we denote $s^{*}=\left\{s_{i} \mid 0 \leq\right.$ $i \leq n\}$.

Definition 3 (Concatenation of paths) If $a=\left(a_{0}, \ldots, a_{n}\right)$ and $b=\left(b_{0}, \ldots, b_{n}\right)$ are two $n$-paths such that $a_{n}$ is $n$-adjacent or equal to $b_{0}$ then we denote $a . b=$ $\left(a_{0}, \ldots, a_{n}, b_{o}, \ldots, b_{m}\right)$, which is called the concatenation of the two paths a and $b$.

\subsection{Homotopy of paths, Fundamental Group}

The fundamental group in digital topology, as defined by Kong in [3], has shown to be a very useful tool for studying topology of digital sets. Here, we define an analogue to the fundamental group for digital surfaces. First, we must define a relation of elementary deformation between paths, the elementary $\mathcal{H}$-deformation relation. 
Definition 4 (elementary $\mathcal{H}$-deformation and homotopy of paths) Let $X \subset \Sigma$. Let $c=\left(x_{i}\right)_{i=0, \ldots, q}$ and $c^{\prime}=\left(x_{i}^{\prime}\right)_{i=0, \ldots, q^{\prime}}$ be two $v$-paths in $X$. The path $c$ is said to be an elementary $\mathcal{H}$-deformation of $c^{\prime}$ in $X$ if $c=\pi_{1} \cdot \alpha . \pi_{2}, c^{\prime}=\pi_{1} \cdot \alpha^{\prime} . \pi_{2}$. Where $\alpha$ and $\alpha^{\prime}$ have the same extremities and are both contained in a common loop of the surface $\Sigma$ (note that the path $\pi_{1}$ or $\pi_{2}$ can be empty).

We define the n-homotopy relation between paths as the transitive closure of the elementary $\mathcal{H}$-deformation: two n-paths $c$ and $c^{\prime}$ are called $n$-homotopic (which we denote by $c \simeq_{n} c^{\prime}$ ) if there exists a sequence of $\mathcal{H}$-deformations which allows us to obtain one from the other.

Remark 1 If a path $c$ is not closed, then any path $c^{\prime}$ which is $n$-homotopic to $c$ must have the same extremities as $c$.

Now, the set of equivalence classes of closed $n$-paths from a surfel $b$ (called the base point) to $b$ in $\Sigma$, given an operation on classes induced by concatenation of paths, has a group structure. It is called the fundamental group of $\Sigma$ with base point $b$ and denoted by $\Pi_{n}(b, \Sigma)$. This (generally non-abelian) group structure is very useful for studying the topology of objects in the digital spaces $\mathrm{Z}^{2}$ and $\mathrm{Z}^{3}$ or surfaces. It is used for example for the characterization of topology preservation (1] and [5]).

This first and simple definition of elementary deformation of paths will be too "large" in our context and proofs in the sequel will need to consider a more restrictive transformation for $v$-paths with simpler conditions, which leads to the same notion of homotopy.

Definition 5 Let $X \subset \Sigma, c=\left(x_{i}\right)_{i=0, \ldots, q}$ and $c^{\prime}=\left(x_{i}^{\prime}\right)_{i=0, \ldots, q^{\prime}}$ be two $v$-paths in $X$. Then, $c$ is said to be an elementary $\mathcal{L}$-deformation of $c^{\prime}$ in $X$ if $c=$ $\pi_{1} \cdot\left(s_{1}, s_{2}, s_{3}\right) \cdot \pi_{2}$ and $c^{\prime}=\pi_{1} \cdot\left(s_{1}, s_{2}\right) \cdot \pi_{2}$. Where the three surfels $s_{1}, s_{2}$ and $s_{3}$ belong to a common loop of $\Sigma$. We define the $\mathcal{L}$-deformation as the symmetric and transitive closure of the elementary $\mathcal{L}$-deformation.

In other words, the elementary $\mathcal{L}$-relation links two $v$-paths which are almost the same except that one is obtained by deletion in the other of a surfel which belongs to the same loop as its predecessor and successor. Now, we can state the following property :

Proposition 1 Two $v$-paths $c$ and $c^{\prime}$ are $v$-homotopic in a surface $X \subset \Sigma$ if and only if one is an $\mathcal{L}$-deformation of each other in $X$.

\section{The intersection number : Definition}

In the following, paths all lie on a digital surface $\Sigma$. If a path is closed, the subscripts of surfels must be read modulo $l$ where $l$ is the length of the path.

Notation 2 (Vertices and oriented edges) Since a surfel has four vertices, we can order these vertices as in [6] by distinguishing one vertex for each type 
of surfel (we distinguish 6 types of surfels according to the outward normal vector) and impose a turning order for vertices around the outward normal to the surfel. Each vertex of a given surfel is associated with a number in $\{0,1,2,3\}$. With this parameterization of vertices we can define oriented edges as couples of consecutive vertices according to the cyclic order. So, for each surfel, we have the four following oriented edges : $(0,1),(1,2),(2,3)$ and $(3,0)$. If $\{a, b\} \subset\{0,1,2,3\}$, we denote as an interval $[a, b]$ the set of vertices met when looking after vertices from $a$ to $b$ in the cyclic order defined before. So, we can write $c \in[a, b]$ if the vertex $c$ is met when looking for vertices from $a$ to $b$. For an $e$-path $\pi=$ $\left(y_{k}\right)_{k=0, \ldots, p}$ and for $k \in\{0, \ldots, p\}$, we define front $_{\pi}(k)$ (resp. back $\left._{\pi}(k)\right)$ as the oriented edge $(a, b)$ with $a, b \in\{0,1,2,3\}$ of the surfel $y_{k}$ shared as an edge by $y_{k}$ and $y_{k+1}$ (resp. $y_{k}$ and $\left.y_{k-1}\right)$. Remark that $b_{a c k}(0)$ and front $_{\pi}(p)$ are not defined if $\pi$ is not closed.

Definition 6 Let $\pi=\left(y_{k}\right)_{k=0, \ldots, p}$ be an $n$-path. We say that $\pi$ has a local back and forth at the surfel $y_{k}$ if $y_{k-1}=y_{k+1}$.

Remark 2 Suppose that $\pi$ has no local back and forth. Then, if $(a, b)=$ back $_{\pi}(k)$ and $(c, d)=$ front $_{\pi}(k)$ then $a \neq c$ and $b \neq d$. Indeed, since the edges are oriented, $a=c$ implies that $b=d$; this would mean that the front and back edges of $y_{k}$ are equal, so that we would have $y_{k-1}=y_{k+1}$.

We want to define locally, at each point of an $e$-path the right side and the left side on the surface, taking into account the orientation of the surface (Definition 8 below).

Definition 7 Let $\pi=\left(y_{k}\right)_{k=0, \ldots, p}$ be an e-path. For $k \in\{1, \ldots, p-1\}(k \in$ $\{0, \ldots, p\}$ if $\pi$ is closed $)$, we define the sets $L_{\pi}(k)$ and $R_{\pi}(k)$ both included in $\{0,1,2,3\}$. Let $(a, b)=$ back $_{\pi}(k)$ and $(c, d)=$ front $_{\pi}(k)$. If $y_{k-1} \neq y_{k+1}$ then we define $L_{\pi}(k)=[d, a]$ and $R_{\pi}(k)=[b, c]$ (see Figure[3). If $y_{k-1}=y_{k+1}$ we define $L_{\pi}(k)=R_{\pi}(k)=\emptyset$. Note that $L_{\pi}(k)$ and $R_{\pi}(k)$ are also defined for $k=0$ or $k=p$ in the case when $\pi$ is a closed $e-$ path.

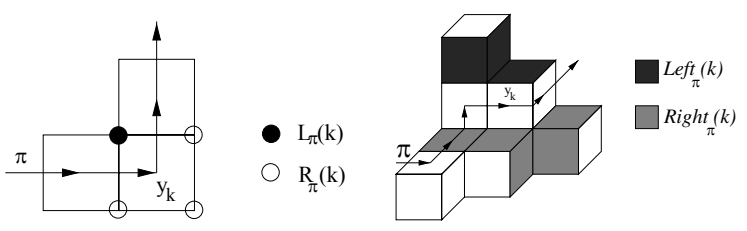

Fig. 3. A surfel $y_{k}$ of $\pi$, and the two sets $L_{\pi}(k)$ and $R_{\pi}(k)$.
Fig. 4. A surfel $y_{k}$ of $\pi$, and the two sets Left $_{\pi}(k)$ and $\operatorname{Right}_{\pi}(k)$.

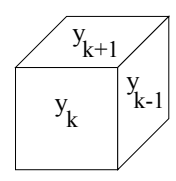

Fig. 5. An example for which $\operatorname{Right}_{\pi}(k)=\emptyset$. 
Notation 3 For a surfel $x$ and a given vertex number $w \in\{0,1,2,3\}$ we denote by $\mathcal{L}_{w}(x)$ the unique loop associated to the vertex $w$ of $x$ which contains the surfel $x$.

Definition 8 Let $\pi=\left(y_{k}\right)_{k=0, \ldots, p}$ be an $e-p a t h$. For $0<k<p(0 \leq k \leq p$ if $\pi$ is closed) we define the set of surfels Left ${ }_{\pi}(k)$ and $\operatorname{Right}_{\pi}(k)$ by:

$$
\begin{gathered}
\operatorname{Left}_{\pi}(k)=\left[\bigcup_{w \in L_{\pi}(k)} \mathcal{L}_{w}\left(y_{k}\right)\right] \backslash\left\{y_{k-1}, y_{k}, y_{k+1}\right\} \text { and } \\
\operatorname{Right}_{\pi}(k)=\left[\bigcup_{w \in R_{\pi}(k)} \mathcal{L}_{w}\left(y_{k}\right)\right] \backslash\left\{y_{k-1}, y_{k}, y_{k+1}\right\} .
\end{gathered}
$$

Note that one of these two sets can be empty if $\left\{y_{k-1}, y_{k}, y_{k+1}\right\}$ constitute a loop with a length 3 (i.e. $y_{k-1}$ is e-adjacent to $y_{k+1}$ ). Such a case is depicted by Figure 5 In the case when $y_{k-1}=y_{k+1}$, both sets Left ${ }_{\pi}(k)$ and Right ${ }_{\pi}(k)$ will be empty since $L_{\pi}(k)$ and $R_{\pi}(k)$ have been defined as empty in this case. See Figure 4 for an example of such sets.

Remark 3 Due to our assumption that loops are topological disks, we have:

$$
\operatorname{Left}_{\pi}(k) \cap \operatorname{Right}_{\pi}(k)=\emptyset .
$$

If $y_{k-1}$ is not $e$-adjacent to $y_{k+1}$ then $N_{v}\left(y_{k}\right) \backslash\left\{y_{k-1}, y_{k+1}\right\}$ has exactly two $e_{y_{k}}$-connected components (see Definition 1): $\operatorname{Right}_{\pi}(k)$ and $\operatorname{Left}_{\pi}(k)$.

In the sequel of this paper we have set some restrictions on the paths studied. In this goal, we define a property $\mathcal{P}$.

Notation 4 Let $c=\left(x_{i}\right)_{i=0, \ldots, q}$ be a $v$-path and $\pi=\left(y_{k}\right)_{k=0, \ldots, p}$ be an $e-p a t h$ in $\Sigma$. We say that the property $\mathcal{P}(\pi, c)$ is satisfied if when $c$ is not closed then $c_{0}, c_{q} \notin \pi^{*}$ and when $\pi$ is not closed then $y_{0}, y_{p} \notin c^{*}$.

Definition 9 Given a v-path $c=\left(x_{i}\right)_{i=0, \ldots, q}$ and an e-path $\pi=\left(y_{k}\right)_{k=0, \ldots, p}$ such that $\mathcal{P}(\pi, c)$ holds, we introduce the contribution to the intersection number of a couple $(k, i)$ with $k \in\{1, \ldots, p-1\} \quad(\{0, \ldots, p\}$ if $\pi$ is closed) and $i \in$ $\{1, \ldots, q-1\} \quad(\{0, \ldots, q\}$ if $c$ is closed $)$ by $\mathcal{I}_{\pi, c}(k, i)=\mathcal{I}_{\pi, c}^{-}(k, i)+\mathcal{I}_{\pi, c}^{+}(k, i)$ where:

$$
\begin{aligned}
& \mathcal{I}_{\pi, c}^{-}(k, i)=\frac{1}{2} \text { if } x_{i}=y_{k}, x_{i-1} \in \operatorname{Right}_{\pi}(k) . \mathcal{I}_{\pi, c}^{+}(k, i)=-\frac{1}{2} \text { if } x_{i}=y_{k}, x_{i+1} \in \operatorname{Right}_{\pi}(k) . \\
& \mathcal{I}_{\pi, c}^{-}(k, i)=-\frac{1}{2} \text { if } x_{i}=y_{k}, x_{i-1} \in \operatorname{Left}_{\pi}(k) . \mathcal{I}_{\pi, c}^{+}(k, i)=\frac{1}{2} \text { if } x_{i}=y_{k}, x_{i+1} \in \text { Left }_{\pi}(k) . \\
& \mathcal{I}_{\pi, c}^{-}(k, i)=0 \text { in all other cases. } \\
& \mathcal{I}_{\pi, c}^{+}(k, i)=0 \text { in all other cases. }
\end{aligned}
$$

Note that $\mathcal{I}_{\pi, c}^{-}(k, i)=0$ (resp. $\left.\mathcal{I}_{\pi, c}^{+}(k, i)=0\right)$ if $x_{i} \neq y_{k}$ or $y_{k-1}=y_{k+1}$ or $x_{i-1} \in\left\{y_{k-1}, y_{k}, y_{k+1}\right\}$ (resp. $x_{i+1} \in\left\{y_{k-1}, y_{k}, y_{k+1}\right\}$ ). If $y_{k-1}=y_{k+1}$, we have $\mathcal{I}_{\pi, c}^{-}(k, i)=\mathcal{I}_{\pi, c}^{+}(k, i)=0$ since $\operatorname{Left}_{\pi}(k)=\operatorname{Right}_{\pi}(k)=\emptyset$.

In other words, $\mathcal{I}_{\pi, c}^{-}(k, i)$ depends on the position of $x_{i-1}$ relative to the e-path $\pi$ at the surfel $y_{k}$. And $\mathcal{I}_{\pi, c}^{+}(k, i)$ depends on the position of $x_{i+1}$. 


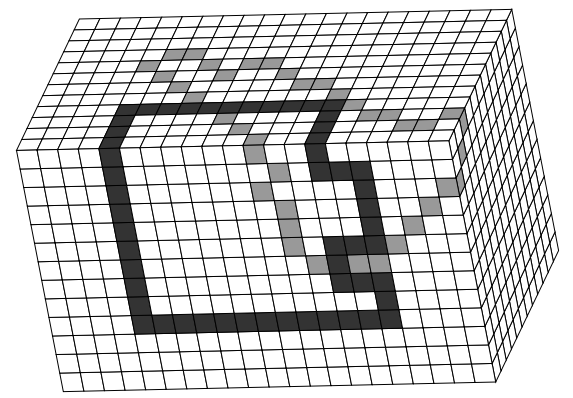

Fig. 6. A $v$-path $c$ (light grey) and an $e$-path $\pi$ (in dark grey) such that $\mathcal{I}_{\pi, c}=0$.

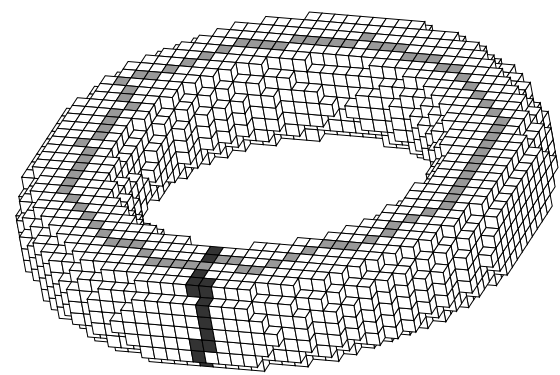

Fig. 7. A $v$-path $c$ and an $e$-path $\pi$ such that $\mathcal{I}_{\pi, c}= \pm 1$.

Definition 10 Let $c=\left(x_{i}\right)_{i=0, \ldots, q}$ be a $v$-path and let $\pi=\left(y_{k}\right)_{k=0, \ldots, p}$ be an e-path satisfying the property $\mathcal{P}(\pi, c)$. The intersection number of the $v$-path $c$ and the $e-$ path $\pi$, denoted by $\mathcal{I}_{\pi, c}$ is defined by:

$$
\mathcal{I}_{\pi, c}=\sum_{k=0}^{p-1} \sum_{i=0}^{q-1} \mathcal{I}_{\pi, c}(k, i)=\sum_{k=0}^{p-1} \sum_{i \mid x_{i}=y_{k}} \mathcal{I}_{\pi, c}(k, i)=\sum_{i=0}^{q-1} \sum_{k \mid x_{i}=y_{k}} \mathcal{I}_{\pi, c}(k, i) .
$$

Figure 6] and Figure 7 show two examples of intersection numbers.

Notation 5 We denote:

$$
\mathcal{I}_{\pi, c}^{\pi}(i)=\sum_{k=0}^{p-1} \mathcal{I}_{\pi, c}(k, i) \text { and } \mathcal{I}_{\pi, c}^{c}(k)=\sum_{i=0}^{q-1} \mathcal{I}_{\pi, c}(k, i)
$$

\section{Independence up to homotopy}

The purpose of this section is to state and sketch the proof of the two following theorems:

Theorem 1 Let $\pi=\left(y_{k}\right)_{k=0, \ldots, p}$ be an e-path, $c=\left(x_{i}\right)_{i=0, \ldots, q}$ and $c^{\prime}=$ $\left(x_{i}^{\prime}\right)_{i=0, \ldots, q^{\prime}}$ be two $v$-paths on a digital surface $\Sigma$ such that $\mathcal{P}(\pi, c)$ and $\mathcal{P}\left(\pi, c^{\prime}\right)$ hold. If $c^{\prime}$ is $v$-homotopic to $c$ in $\Sigma$ (in $\Sigma \backslash\left\{y_{0}, y_{p}\right\}$ if $\pi$ is not closed), then $\mathcal{I}_{\pi, c}=\mathcal{I}_{\pi, c^{\prime}}$.

Theorem 2 Let $c=\left(x_{i}\right)_{i=0, \ldots, q}$ be a v-path of a digital surface $\Sigma, \pi=$ $\left(y_{k}\right)_{k=0, \ldots, p}$ and $\pi^{\prime}=\left(y_{k}^{\prime}\right)_{k=0, \ldots, p^{\prime}}$ be two $e-p a t h s$ such that $\mathcal{P}(\pi, c)$ and $\mathcal{P}\left(\pi^{\prime}, c\right)$ hold. If $\pi^{\prime}$ and $\pi$ are e-homotopic in $\Sigma$ (in $\Sigma \backslash\left\{x_{0}, x_{q}\right\}$ if $c$ is not closed), then $\mathcal{I}_{\pi, c}=\mathcal{I}_{\pi^{\prime}, c}$. 
We first prove Theorem 1, and Theorem 2 will appear as a corollary of Theorem 1 .

Since $v$-homotopy and $\mathcal{L}$-deformation are equivalent relations, then we can prove the equality when considering the latter relation between the paths $c$ and $c^{\prime}$. Furthermore, to prove these properties for any $\mathcal{L}$-deformation, it is sufficient to prove it for an elementary $\mathcal{L}$-deformation. Now, in order to prove this equality, we consider the contribution of intersection intervals between the two paths $\pi$ and $c$ on a first hand, $\pi$ and $c^{\prime}$ on the other hand. But these intervals can have very bad properties when considering the general case, especially when $\pi$ has local back an forths (see Definition 6). So, to avoid this special cases, we first suppose that the path $\pi$ has no local back and forth and prove Theorem 1 in this case. In a second step, we prove that the intersection number between a $v$-path $c$ and an $e$-path $\pi$ is left unchanged when one removes all back and forths in $\pi$.

First we state some technical lemmas.

\subsection{Important lemmas}

Let $\pi=\left(y_{k}\right)_{k=0, \ldots, p}$ be an $e$-path with no local back and forth on $\Sigma$, then we have the three following lemmas:

Lemma 2 Let $k \in[1, p-1]$ if $\pi$ is not closed and $k \in[0, p]$ otherwise. If $x_{1}$ and $x_{2}$ are two surfels of a given loop containing the surfel $y_{k}$ such that $\left\{x_{1}, x_{2}\right\} \cap\left\{y_{k-1}, y_{k}, y_{k+1}\right\}=\emptyset$, then we have either $\left\{x_{1}, x_{2}\right\} \subset$ Left $_{\pi}(k)$ or $\left\{x_{1}, x_{2}\right\} \subset \operatorname{Right}_{\pi}(k)$.

Lemma 3 Let $k \in[1, p-2]$ if $\pi$ is not closed and $k \in[0, p-1]$ otherwise. If $x$ is a surfel such that $x \notin\left\{y_{k-1}, y_{k}, y_{k+1}, y_{k+2}\right\}$ and $x, y_{k}$ and $y_{k+1}$ are included in a common loop, then: $x \in$ Right $_{\pi}(k)$ (resp. Left $\left._{\pi}(k)\right)$ if and only if $x \in \operatorname{Right}_{\pi}(k+1)$ (resp. Left $\left.t_{\pi}(k+1)\right)$.

Lemma 4 Let $k \in[1, p-3]$ if $\pi$ is not closed and $k \in[0, p-2]$ otherwise. If $k$ is such that $y_{k}, y_{k+1}$ and $y_{k+2}$ are included in a common loop, $y_{k-1} \neq y_{k+2}$ and $y_{k+3} \neq y_{k}$, then $y_{k+2} \in \operatorname{Right}_{\pi}(k)$ (resp. Left $t_{\pi}(k)$ ) if and only if $y_{k} \in$ $\operatorname{Right}_{\pi}(k+2)\left(\right.$ resp. Left $\left._{\pi}(k+2)\right)$.

\subsection{Independence when $\pi$ has no local back and forth}

In order to prove the Theorem [1] we use the following proposition:

Proposition 5 Let $c=\left(x_{i}\right)_{i=0, \ldots, q}$ and $c^{\prime}=\left(x_{i}^{\prime}\right)_{i=0, \ldots, q^{\prime}}$ be two $v$-paths on a digital surface $\Sigma$, let $\pi=\left(y_{k}\right)_{k=0, \ldots, p}$ be an e-path with no local back and forth such that $\mathcal{P}(\pi, c)$ and $\mathcal{P}\left(\pi, c^{\prime}\right)$ hold. If $c^{\prime}$ is an elementary $\mathcal{L}$-deformation of $c$ in $\Sigma$ (in $\Sigma \backslash\left\{y_{0}, y_{p}\right\}$ if $\pi$ is not closed) then $\mathcal{I}_{\pi, c^{\prime}}=\mathcal{I}_{\pi, c}$.

In the sequel of this subsection, $c$ and $c^{\prime}$ are $v$-paths satisfying the hypothesis of Proposition 5] From the very definition of the elementary $\mathcal{L}$-deformation, $c^{\prime}$ is obtained by the removal in $c$ of the surfel $x_{l}(0<l<q$ if $c$ is not closed, 
and $0 \leq l \leq q$ otherwise). This means that $c^{\prime}=\left(x_{0}^{\prime}, \ldots, x_{q-1}^{\prime}\right)$ where $x_{i}^{\prime}=x_{i}$ if $0 \leq i<l$ and $x_{i}^{\prime}=x_{i+1}$ if $l \leq i<q$.

We have to prove that $\mathcal{I}_{\pi, c}=\mathcal{I}_{\pi, c^{\prime}}$ with $\mathcal{I}_{\pi, c}=\sum_{i=0}^{q-1} \mathcal{I}_{\pi, c}^{\pi}(i)$ and $\mathcal{I}_{\pi, c^{\prime}}=\sum_{i=0}^{q-2} \mathcal{I}_{\pi, c^{\prime}}^{\pi}(i)$.

For $i=0, \ldots, l-2$ if $c$ is closed and for $i=1, \ldots, l-2$ otherwise, we have $x_{i-1}=x_{i-1}^{\prime}, x_{i}=x_{i}^{\prime}$ and $x_{i+1}=x_{i+1}^{\prime}$, so that $\mathcal{I}_{\pi, c^{\prime}}^{\pi}(i)=\mathcal{I}_{\pi, c}^{\pi}(i)$. If $c$ is not closed then $\mathcal{I}_{\pi, c^{\prime}}^{\pi}(0)=\mathcal{I}_{\pi, c}^{\pi}(0)=0$ since $x_{0}=x_{0}^{\prime} \notin \pi^{*}$. Similarly, for $i=l+1, \ldots, q-2$ we have $x_{i-1}^{\prime}=x_{i}, x_{i}^{\prime}=x_{i+1}$ and $x_{i+1}^{\prime}=x_{i+2}$, so that $\mathcal{I}_{\pi, c^{\prime}}^{\pi}(i)=\mathcal{I}_{\pi, c}^{\pi}(i+1)$.

Finally we have to evaluate the difference :

$$
\mathcal{I}_{\pi, c}-\mathcal{I}_{\pi, c^{\prime}}=\left[\mathcal{I}_{\pi, c}^{\pi}(l-1)+\mathcal{I}_{\pi, c}^{\pi}(l)+\mathcal{I}_{\pi, c}^{\pi}(l+1)\right]-\left[\mathcal{I}_{\pi, c^{\prime}}^{\pi}(l-1)+\mathcal{I}_{\pi, c^{\prime}}^{\pi}(l)\right]
$$

In order to prove that this difference is zero, we sum the contributions of intersection intervals between $\pi$ and the three surfels $\left\{x_{l-1}, x_{l}, x_{l+1}\right\}$ of $c$ on a first hand, and between $\pi$ and the two surfels $\left\{x_{l-1}^{\prime}, x_{l}^{\prime}\right\}$ of $c^{\prime}$ on the other hand.

Definition 11 Let $b=\left(z_{0}, \ldots, z_{r}\right)$ be an e-path, and $d=\left(t_{0}, \ldots, t_{s}\right)$ be $a$ $v$-path. Let $X$ be a set of integers in $[0, s]$ ( $X$ will be either $\{l-1, l, l+1\}$ or $\{l-1, l\})$. We define the set of intersection intervals between the path $b$ and $d$ in $X$ by:

$$
\Lambda_{b, d}(X)=\left\{\left[k_{1}, k_{2}\right] \mid \forall k \in\left[k_{1}, k_{2}\right], \exists h \in X, z_{k}=t_{h} \text { and } \forall i \in X, t_{i} \notin\left\{z_{k_{1}-1}, z_{k_{2}+1}\right\}\right\}
$$

When applying this definition, we shall use $b=\pi$ and $d=c$ or $d=c^{\prime}$.

In other words, an interval $\left[k_{1}, k_{2}\right]$ is a maximal sequence of subscripts of surfels of $\pi$ which belong to a certain subset of $c^{*}$. The idea of the following is that the deletion of a surfel in $c$ will either suppress, reduce or disconnect such intersection intervals but the sum of the contributions of the resulting intervals will be equal to the contribution of the initial interval. More precisely, we shall prove:

Proposition 6 Denoting $\Lambda=\Lambda_{\pi, c}(\{l-1, l, l+1\})$ and $\Lambda^{\prime}=\Lambda_{\pi, c^{\prime}}(\{l-1, l\})$, then $\forall \lambda \in \Lambda$ :

$$
\sum_{k \in \lambda} \mathcal{I}_{\pi, c}(k, l-1)+\mathcal{I}_{\pi, c}(k, l)+\mathcal{I}_{\pi, c}(k, l+1)=\sum_{\substack{\lambda^{\prime} \in \Lambda^{\prime} \\ \lambda^{\prime} \subset \lambda}} \sum_{k \in \lambda^{\prime}} \mathcal{I}_{\pi, c^{\prime}}(k, l-1)+\mathcal{I}_{\pi, c^{\prime}}(k, l)
$$

Sketch of proof: To prove this proposition, we first note that the intersection intervals in $\Lambda$ must have a length $l \leq 3$ or the three surfels $x_{l-1}, x_{l}$ and $x_{l+1}$ must be pairwise $e$-adjacent as in the only two possible configurations depicted in Figure 8 The equality of Proposition 6 is shown by using Lemma 2 for intervals whith a length 1, Lemma 3 for intervals with a length 2 and Lemma 4 for intervals with a length 3 . For intervals with a length greater then 3 , the proof uses these three lemmas and considerations about periodicity of $\pi$ in this special case. 

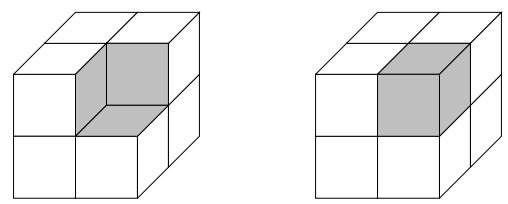

Fig. 8. Two cases when 3 surfels are pairwise $e$-adjacent.

\section{Sketch of proof of Proposition [5:}

Since $\left\{k \in[0, p] \mid y_{k} \in\left\{x_{l-1}, x_{l}, x_{l+1}\right\}\right\}=\bigcup_{\lambda \in \Lambda} \lambda$ and the $\lambda \in \Lambda$ are pairwise disjoint, and similarly for $\Lambda^{\prime}$, due to equation (1) and Proposition 6] we have:

$$
\begin{aligned}
& \sum_{\lambda \in \Lambda} \sum_{k \in \lambda}^{\mathcal{I}_{\pi, c}-\mathcal{I}_{\pi, c^{\prime}}=} \mathcal{I}_{\pi, c}(k, l-1)+\mathcal{I}_{\pi, c}(k, l)+\mathcal{I}_{\pi, c}(k, l+1)-\sum_{\lambda \in \Lambda} \sum_{\frac{\lambda^{\prime} \in \Lambda^{\prime}}{\lambda^{\prime} \subset \lambda}} \sum_{k \in \lambda^{\prime}} \mathcal{I}_{\pi, c^{\prime}}(k, l-1)+\mathcal{I}_{\pi, c^{\prime}}(k, l) \\
& =0 .
\end{aligned}
$$

\subsection{Independence under Shrunk operation}

Given an $e$-path $\pi=\left(y_{k}\right)_{k=0, \ldots, p}$, we define the operations $\operatorname{shrunk}(\pi)$ and $\operatorname{Shrunk}(\pi)$ :

Definition 12 Let $\pi=\left(y_{k}\right)_{k=0, \ldots, p}$ be an e-path such that that there exists $l$, $0<l<p\left(0 \leq l \leq p\right.$ if $\pi$ is closed) such that $y_{l-1}=y_{l+1}$ and for all $l^{\prime}$ such that $y_{l^{\prime}-1}=y_{l^{\prime}+1}$, then $l<l^{\prime}$. We define shrunk $(\pi)$ as the path obtained by the deletion in $\pi$ of the two surfels $y_{l}$ and $y_{l+1}$. Remark that shrunk $(\pi)$ is still an $e-$ path since $y_{l-1}\left(=y_{l+1}\right)$ is e-adjacent to $y_{l+2}$.

Shrunk $(\pi)$ is the path obtained after sequential applications of shrunk on $\pi$ until the resulting path has no local back and forth.

The following lemma means that removing all back and forths in the $e$-path leaves the intersection number unchanged.

Lemma 7 Let $\pi=\left(y_{k}\right)_{k=0, \ldots, p}$ be an $e-p a t h$ on $\Sigma$ and $c=\left(x_{i}\right)_{i=0, \ldots, q}$ a v-path on $\Sigma$ such that $\mathcal{P}(\pi, c)$ holds then $\mathcal{I}_{\pi, c}=\mathcal{I}_{\text {Shrunk }(\pi), c}$.

In order to prove this lemma, it is sufficient to prove that $\mathcal{I}_{\pi, c}=\mathcal{I}_{\text {shrunk }(\pi), c}$. To to this, we first observe that if $\pi$ has its first local back and forth at $y_{l}$ $\left(l \in[0, p]\right.$ if $\pi$ is closed, $l \in[1, p-1]$ otherwise) then $\mathcal{I}_{\pi, c}-\mathcal{I}_{\operatorname{Shrunk}(\pi), c}=$ $\mathcal{I}_{\pi, c}^{c}(l-1)+\mathcal{I}_{\pi, c}^{c}(l)+\mathcal{I}_{\pi, c}^{c}(l+1)-\mathcal{I}_{\text {shrunk }(\pi), c}^{c}(l-1)$. Then, we can prove for $i=0, \ldots, q-1$ that $\mathcal{I}_{\pi, c}(l-1, i)+\mathcal{I}_{\pi, c}(l, i)+\mathcal{I}_{\pi, c}(l+1, i)-\mathcal{I}_{\text {shrunk }(\pi), c}(l-1, i)=0$. Several cases must be examined depending on the position of surfels $x_{i}, x_{i-1}$ and $x_{i+1}$ relative to the surfels $y_{l-2}, y_{l-1}=y_{l+1}, y_{l}$ and $y_{l+2}$. Each case is straightforward. 


\subsection{Proofs of the main results}

Proof of Theorem 1; By induction, it is sufficient to prove Theorem [1 in the case when $c^{\prime}$ is an elementary $\mathcal{L}$-deformation of $c$. If $\pi^{\prime}=\operatorname{Shrunk}(\pi)$, then lemma 7 shows that $\mathcal{I}_{\pi, c}=\mathcal{I}_{\pi^{\prime}, c}$. Since $\pi^{\prime}$ has no local back and forth, and since $c^{\prime}$ is an elementary $\mathcal{L}$-deformation of $c^{\prime}$ in $\Sigma$ (in $\Sigma \backslash\left\{y_{0}, y_{p}\right\}$ if $\pi$ is not closed), then from Proposition 5 we have : $\mathcal{I}_{\pi^{\prime}, c}=\mathcal{I}_{\pi^{\prime}, c^{\prime}}$. Now, Lemma 7 implies that $\mathcal{I}_{\text {Shrunk }(\pi), c^{\prime}}=\mathcal{I}_{\pi, c^{\prime}}$. Finally, $\mathcal{I}_{\pi, c}=\mathcal{I}_{\pi, c^{\prime}}$.

Sketch of proof of Theorem 2, It is sufficient to prove Theorem 2] in the case when $\pi^{\prime}$ is an elementary $\mathcal{H}$-deformation of $\pi$. Hence we assume $\pi=\pi_{1} . \alpha . \pi_{2}$ and $\pi^{\prime}=\pi_{1} \cdot \alpha^{\prime} \cdot \pi_{2}$ with $\alpha$ and $\alpha^{\prime}$ being two $e$-paths with same extremities and being contained in a loop $\mathcal{L}$.

Then, we prove that $c$ is $v$-homotopic to a $v$-path $c^{\prime}$ which contains no surfel of the loop $\mathcal{L}$ so that $\mathcal{I}_{\pi, c^{\prime}}=\mathcal{I}_{\pi^{\prime}, c^{\prime}}$. From Theorem 1 we have $\mathcal{I}_{\pi, c}=$ $\mathcal{I}_{\pi, c^{\prime}}=\mathcal{I}_{\pi^{\prime}, c^{\prime}}=\mathcal{I}_{\pi^{\prime}, c}$.

\section{A new Jordan theorem}

Definition $13 A$ simple closed $n$-curve of surfels in $\Sigma$ is an $n$-connected set $\mathcal{C}$ of surfels such that for any $x \in \mathcal{C}$, the surfel $x$ has exactly two $n$-neighbors in $\mathrm{C}$.

Definition 14 For a simple closed $n$-curve $\mathcal{C}$ there exists a sequence $c=$ $\left(x_{i}\right)_{i=0, \ldots, q}$ with $q=\operatorname{Card}(\mathcal{C})$ such that for $i, j \in[0, q]$ we have $x_{i}, x_{j} \in \mathcal{C}$, and such that $x_{i}$ is $n$-adjacent to $x_{j}$ if and only if $i=j+1[q]$ or $i=j-1[q]$.

Such a closed path $c$ is called a parametrization of $\mathcal{C}$.

We shall use the following theorem:

Theorem 3 (see [7]) A digital surface is an e-connected set of surfel.

Using the notion of the intersection number, we prove:

Theorem 4 Let $(n, \bar{n}) \in\{(e, v),(v, e)\}$. If $\pi=\left(y_{k}\right)_{k=0, \ldots, p}$ is a parametrization of a simple closed $n$-curve of surfels on a digital surface $\Sigma$, not included in a loop, such that $\pi \simeq_{n} \pi_{0}=(y, y)$ ( $\pi$ is $n$-homotopic to a path reduced to a single surfel $y)$, then $\Sigma \backslash \pi^{*}$ has exactly two $\bar{n}$-connected components.

In the sequel of this section, $\pi=\left(y_{k}\right)_{k=0, \ldots, p}$ satisfies the hypothesis of Theorem 4. Moreover, to improve the readability, we assume that $n=e$. The idea of the proof is the same in the case $n=v$. We need the two following lemmas :

Lemma 8 For any surfel $y_{k}$ of $\pi$ there exists two surfels $\alpha$ and $\beta$ such that $\alpha \in \operatorname{Right}_{\pi}(k)$ and $\beta \in \operatorname{Left}_{\pi}(k)$.

Lemma 8 is a consequence of the fact that $\pi$ is not contained in a loop. 
Lemma 9 There exists two surfels $\alpha$ and $\beta$ in $\Sigma \backslash \pi^{*}$ which are $v$-adjacent to a surfel $y_{k}$ of $\pi$ and which are not $v$-connected in $\Sigma \backslash \pi^{*}$.

Proof: Let $\alpha$ and $\beta$ be the two surfels defined in Lemma 8 . Now, we suppose by contraposition the existence of a $v$-path $c=\left(x_{i}\right)_{i=0, \ldots, q}$ in $\Sigma \backslash \pi^{*}$ between the surfels $\alpha$ and $\beta, v$-neighbors of the surfel $y_{k}$ in $\pi$ and which does not intersect $\pi^{*}$. We denote $c^{\prime}=\left(x_{0}=\alpha, \ldots, x_{q}=\beta, y_{k}, \alpha\right)$ which is a closed $v$-path. Then, from the definition of $\alpha$ and $\beta, \mathcal{I}_{\pi, c^{\prime}}= \pm 1$. Now, since $\pi$ is $e$-homotopic to a single surfel and from Theorem 2 we should have $\mathcal{I}_{\pi, c^{\prime}}=0$. This contradicts the existence of $\pi$.

Lemma 10 Let $y_{k}$ and $y_{k+1}$ be two consecutive surfels of $\pi$. For any surfel $s \notin \pi^{*}$, which is $v$-adjacent to $y_{k}$, there exists a surfel $t \notin \pi^{*}, v$-adjacent to $y_{k+1}$, and a $v$-path from $s$ to $t$ in $\Sigma \backslash \pi^{*}$.

This lemma can be proved by local considerations.

Proof of theorem 4: From lemma 9 there exists two surfels $\alpha$ and $\beta$ which are $v$-adjacent to a surfel $y_{k}$ and not $v$-connected in $\Sigma \backslash \pi^{*}$. In particular, $\Sigma \backslash \pi^{*}$ has at least two $v$-connected components.

Furthermore, for any surfel $x \in \Sigma \backslash \pi^{*}$, since $\Sigma$ is $e$-connected, then there exists a $v$-path $c^{\prime}$ in $\Sigma \backslash \pi^{*}$ from $x$ to a surfel which is $v$-adjacent to a surfel of $\pi^{*}$.

Using inductively lemma 10, we see that we can prolong the $v$-path $c^{\prime}$ to a $v$-path in $\Sigma \backslash \pi^{*}$ from $x$ to $\alpha$ or from $x$ to $\beta$. This implies that $\Sigma \backslash \pi^{*}$ has at most two $v$-connected components.

\section{Conclusion}

We have defined the intersection number between a $v$-path and an $e$-path lying on a digital surface, and we have proved that this number of "real" intersections between two surfels paths is invariant under homotopic deformations of the two paths. The intersection number is a new "topological invariant" in the context of digital surfaces. Thus, the intersection number has been used to easily prove a new Jordan theorem for surfels curves. It appears to be a useful tool for digital topology. In further work, we shall use the intersection number to study topology preservation in digital surfaces, following the work of [5].

\section{References}

1. G. Bertrand. Simple points, topological numbers and geodesic neighborhoods in cubics grids. Patterns Recognition Letters, 15:1003-1011, 1994.

2. T.J. Fan, G. Medioni, and R. Nevata. Recognising 3d objects using surface descriptions. IEEE Transactions on Pattern Analysis and Machine Intelligence, 1111:11401157, 1989.

3. T. Y. Kong. A Digital Fundamental Group, volume 13. 1989. 
4. A. Lenoir. Fast estimation of mean curvature on the surface of a 3d discrete object. In Proceedings of DGCI'97, Lecture Notes in Computer Science, volume 1347, pages 213-222, 1997.

5. R. Malgouyres and A. Lenoir. Topology preservation within digital surfaces. $M a-$ chine Graphics and Vision, 7(1/2):417-426, 1998. Proceeding of the Computer Graphics and Image Processing.

6. A. Rosenfeld, T.Y. Kong, and A.Y. Wu. Digital surfaces. CVGIP: Graphical Models and Image Processing, 53(4):305-312, 1991.

7. J. K. Udupa. Multidimensional digital boundaries. CVGIP: Graphical Models and Image Processing, 56:311-323, 1994. 